\title{
Topological states in disordered arrays of dielectric nanoparticles
}

\author{
Ling Lin, ${ }^{1,2}$ Sergey Kruk,${ }^{3}$ Yongguan Ke, ${ }^{1,3}$ Chaohong Lee $\odot,{ }^{1,2, *}$ and Yuri Kivshar $\odot^{3, \dagger}$ \\ ${ }^{1}$ Guangdong Provincial Key Laboratory of Quantum Metrology and Sensing and School of Physics and Astronomy, \\ Sun Yat-Sen University (Zhuhai Campus), Zhuhai 519082, China \\ ${ }^{2}$ State Key Laboratory of Optoelectronic Materials and Technologies, Sun Yat-Sen University (Guangzhou Campus), \\ Guangzhou 510275, China \\ ${ }^{3}$ Nonlinear Physics Centre, Research School of Physics, Australian National University, Canberra ACT 2601, Australia
}

(Received 25 July 2020; revised 14 October 2020; accepted 15 October 2020; published 13 November 2020)

\begin{abstract}
We study the interplay between disorder and topology for localized edge states of light in zigzag arrays of Mie-resonant dielectric nanoparticles. We characterize the topological properties of the array by the winding number that depends on both zigzag angle and spacing between nanoparticles. For equal-spacing nanoparticle arrays, the system may have two values of the winding number, $v=0$ or $v=1$, and it demonstrates localization at the edges even in the presence of disorder, as revealed by experimental observations for finite-length ideal and randomized nanoparticle structures. For staggered-spacing nanoparticle arrays, the system possesses richer topological phases characterized by the winding numbers $v=0, v=1$, or $v=2$, which depend on the averaged zigzag angle and the strength of disorder. In a sharp contrast to the equal-spacing zigzag arrays, the staggeredspacing nanoparticle arrays support two types of topological phase transitions induced by the angle disorder, (i) $v=0 \leftrightarrow v=1$ and (ii) $v=1 \leftrightarrow v=2$. More importantly, the spectrum of the staggered-spacing nanoparticle arrays may remain gapped even in the case of a strong disorder.
\end{abstract}

DOI: 10.1103/PhysRevResearch.2.043233

\section{INTRODUCTION}

Topological photonics emerged recently as a novel platform to realize robust optical circuitry protected against disorder [1]. The initial study of topological effects in photonics was inspired largely by direct analogies with the similar effects in condensed-matter physics predicted and observed for topologically nontrivial energy bands of electrons. In photonics, the concept of topological phases offers nontraditional approaches in the search for innovative designs of advanced photonic devices, as well as introducing novel physical effects and their applications [2]. The existence of spatially localized topological edge states has been predicted and realized in a variety of photonic systems, including topological lasers [3], single-photon transport [4], and topological solitons [5]. The study of disorder in topological systems is very important because robustness against disorder is employed extensively as a direct measure of topologically protected features. Anderson localization provides a well-known effect of disorder in condensed-matter physics [6], which originates from interference of coherent waves, and it has been extended from electronic wave functions to other waves such

\footnotetext{
*lichaoh2@mail.sysu.edu.cn

†yuri.kivshar@anu.edu.au

Published by the American Physical Society under the terms of the Creative Commons Attribution 4.0 International license. Further distribution of this work must maintain attribution to the author(s) and the published article's title, journal citation, and DOI.
}

as electromagnetic waves [7,8], acoustic waves [9], and matter waves $[10,11]$. Strong spatial localization of light due to disorder can be realized in periodic or quasiperiodic dielectric structures, and it is represented by photonic band gaps. For systems with nontrivial topological phases, the interplay of topology-induced spatial localization (i.e., topological edge states) and disorder-induced Anderson localization becomes a very important problem, especially when disorder is no longer weak. Here, we address this important problem by studying the effects of disorder in topologically nontrivial zigzag arrays of coupled subwavelength optical resonators. Many topological photonic systems are based on the waveguide geometries. A zigzag array of coupled resonators has emerged as the first nanoscale system with nontrivial topological properties of light [12], and its topology-driven effects have been observed experimentally, including lasing from exciton-polariton nanoscale cavities [3] and third-harmonic generation from nonlinear arrays of dielectric nanoresonators [13]. Recently, the study of topological photonic systems at subwavelength scale has received much interest, including valley-Hall waveguides [14,15], plasmonic metasurfaces [16], and metamaterials [17].

In this paper, we study the interplay between disorder and topology in the zigzag arrays of resonant dielectric nanoparticles in the regime when this system supports nontrivial topological phases. We consider four types of topological arrays with equidistant and nonequidistant spacing between the particles, as illustrated in Fig. 1, and we analyze the topological phases and disorder-induced topological phase transitions in such arrays. We also present experimental results for finite-extent arrays of Mie-resonant dielectric nanoparticles 


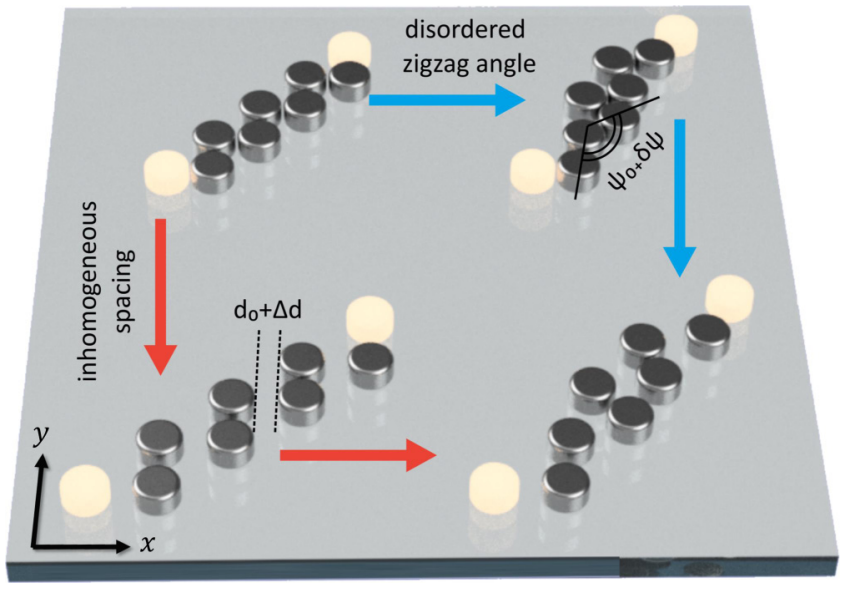

FIG. 1. Schematic diagram of four different types of topologically nontrivial zigzag arrays of dielectric nanoparticles. The Mie-resonant nanodisks are arranged as equal spacing (top) and staggered spacing (bottom), with no disorder in the zigzag angles (left) and with disorder in the zigzag angles (right).

which confirm the basic conclusions on the effect of disorder on topological properties, within the limitation imposed by the finite length of the arrays. Remarkably, for the staggered-spacing arrays, we observe that the topological spectral gap can survive even in the presence of a strong disorder.

The paper is organized as follows. In Sec. II, we introduce our model of a zigzag array of Mie-resonant dielectric nanoparticles, and then in Sec. III we study the topological properties of an ideal (regular) array and its disordered modifications. In Sec. IV, we use the zero-energy localization method to probe the disorder-induced topological transitions. In Sec. V, we generalize our model of the topological zigzag array from the equal-spacing to staggered-spacing nanoparticle arrays. Finally, Sec. VI concludes the paper.

\section{EQUAL-SPACING ZIGZAG ARRAYS}

It was established that topologically nontrivial states may appear in zigzag arrays of nanoparticles with polarizationdependent interaction between electromagnetic modes $[12,13,18,19]$. Here, we follow those earlier predictions and consider the electric or magnetic polarizations of dielectric nanoparticles in the $x-y$ plane, as shown in Fig. 2(a), coupled due to the dipole-dipole interaction. In this experimental platform [13], the disks are large enough to support the first two Mie resonances at around 1500-1600-nm wavelength but are too small to support any significant higher-order multipoles. Thus higher-order multipoles can be neglected at the given disk size and operation wavelength. Such polarization-dependent interaction between two neighboring nanoparticles can be written in the form [19]

$$
V^{\left(j, j^{\prime}\right)}=t_{\|} \vec{e}_{\|}^{\left(j, j^{\prime}\right)} \otimes \vec{e}_{\|}^{\left(j, j^{\prime}\right)}+t_{\perp} \vec{e}_{\perp}^{\left(j, j^{\prime}\right)} \otimes \vec{e}_{\perp}^{\left(j, j^{\prime}\right)},
$$

where $\vec{e}_{\|}^{\left(j, j^{\prime}\right)}$ and $\vec{e}_{\perp}^{\left(j, j^{\prime}\right)}$ denote the identity vectors parallel and perpendicular to the link vector $\vec{r}_{j}-\vec{r}_{j^{\prime}}$, respectively. $V^{\left(j, j^{\prime}\right)}=$ $V^{\left(j^{\prime}, j\right)}$, and the coupling decays with the distance between the
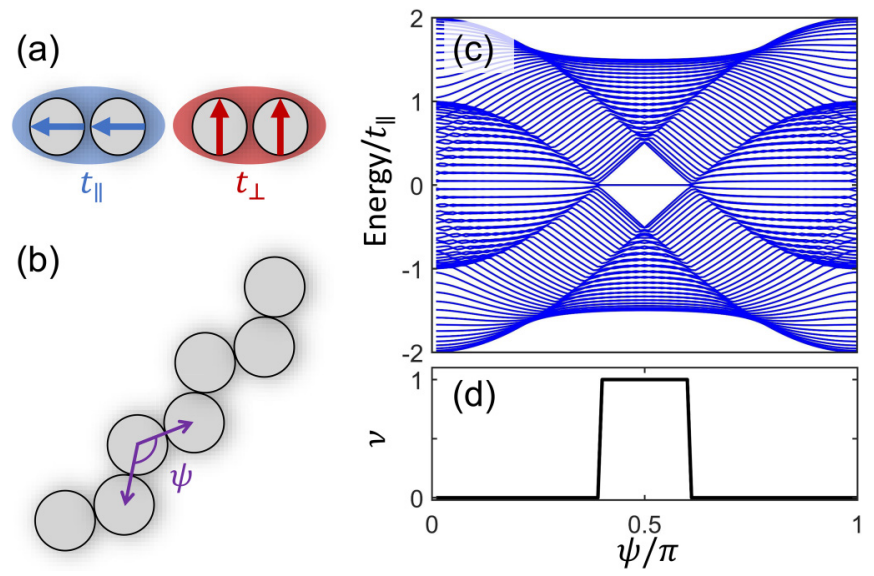

FIG. 2. (a) Illustrations of polarization-dependent dipole interaction between nanoparticles. The arrows inside the particle indicate direction of electric or magnetic polarization. (b) Schematic illustration of the zigzag array. (c) Energy spectrum as a function of zigzag angle $\psi$ under the OBC. (d) Winding number $v$ calculated from Eq. (6).

$j$ th and $j^{\prime}$ th nanoparticles due to the dipole-dipole interaction. It is reasonable to keep only the nearest-neighboring interaction $[12,13,18,19]$.

By decomposing the polarization into $x$ and $y$ modes, the Hamiltonian of such zigzag arrays can be written as

$$
\hat{H}=\sum_{j, v} E_{0} a_{j, v}^{\dagger} a_{j v}+\sum_{j, v, v^{\prime}} a_{j, v}^{\dagger} V_{v, v^{\prime}}^{(j, j+1)} a_{j+1, v^{\prime}}+\text { H.c. },
$$

with

$$
V_{v, v^{\prime}}^{j, j+1}= \begin{cases}t_{\|} \cos ^{2}(\psi / 2)+t_{\perp} \sin ^{2}(\psi / 2), & v=v^{\prime}=x \\ t_{\|} \sin ^{2}(\psi / 2)+t_{\perp} \cos ^{2}(\psi / 2), & v=v^{\prime}=y \\ \left(t_{\|}-t_{\perp}\right) \sin (\psi / 2) \cos (\psi / 2), & v \neq v^{\prime} .\end{cases}
$$

Here, $a_{j, v}^{\dagger}$ and $a_{j, v}$ are the creation and annihilation operators for a $\nu$ mode at the $j$ th nanoparticle, and $\psi$ is the zigzag angle, as indicated in Fig. 2(b). $E_{0}$ is the on-site potential which causes an overall energy shift and hence is neglected. The ratio $t_{\|} / t_{\perp}$ depends on the interaction; for example, $t_{\|} / t_{\perp}=-2$ for the dipole-dipole interaction, and $t_{\|} / t_{\perp}=-4$ for the quadrupole-quadrupole interaction. Here, we consider the case of $t_{\|} / t_{\perp}=-2$.

\section{WINDING NUMBER}

\section{A. Ideal arrays}

We start with the study of an ideal system without disorder. In this case, an infinite system is translationally invariant. Under the periodic boundary condition (PBC), the Bloch Hamiltonian reads [18]

$$
H(K)=\left(\begin{array}{cc}
0 & Q(K) \\
Q^{\dagger}(K) & 0
\end{array}\right),
$$

with

$$
Q(K)=h_{0}+\vec{h}(K) \vec{\sigma} .
$$

Here, $\quad h_{0}=t\left(1+e^{-i K}\right), \quad$ and $\quad \vec{h}(K) \equiv\left(h_{x}, h_{y}, h_{z}\right)=$ $\left[\frac{\Delta}{2} e^{-i K} \sin \psi, 0, \frac{\Delta}{2}\left(1+e^{-i K} \cos \psi\right)\right]$ are components of 

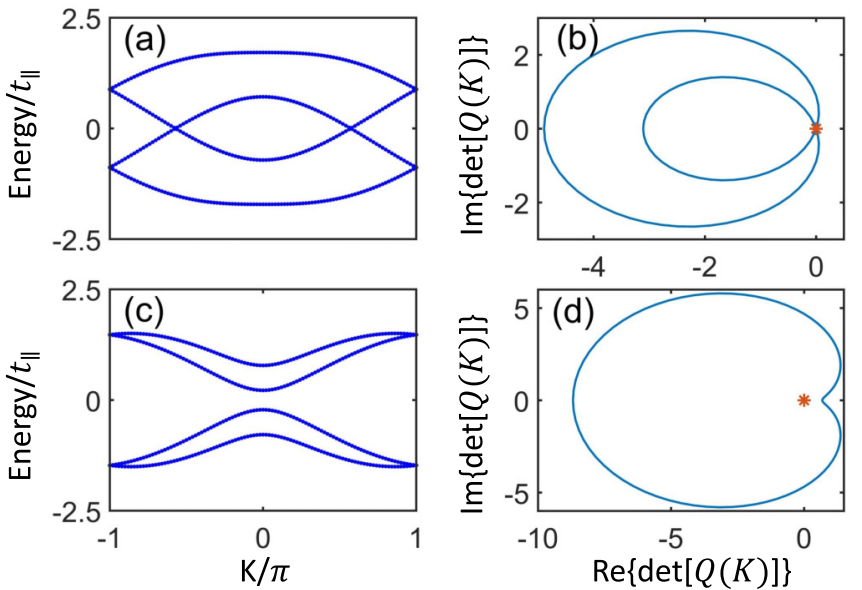

FIG. 3. (a)-(d) Band structure (left column) and det $[Q(K)]$ (right column) for different values of the zigzag angle $\psi$. (a) and (b) $\psi=$ $0.2 \pi$ : the system is gapless, and the curve of $\operatorname{det}[Q(K)]$ lies exactly on the origin. (c) and (d) $\psi=0.44 \pi$ : the system is gapped, and the winding number is well defined.

an effective magnetic field with $t=\left(t_{\|}+t_{\perp}\right) / 2$ and $\Delta=\left(t_{\|}+t_{\perp}\right) . \vec{\sigma} \equiv\left(\sigma_{x}, \sigma_{y}, \sigma_{z}\right)$ are the Pauli matrices.

According to the classification of topological insulators and superconductors, this system belongs to the so-called BDI class [20], meaning a certain "Cartan label" given to the corresponding symmetric space in Élie Cartan's classification scheme dating back to 1926 . The topological invariant of such systems is presented by the winding number,

$$
\begin{aligned}
v & =\frac{1}{2 \pi i} \int_{-\pi}^{\pi} d K \operatorname{Tr}\left[Q^{-1}(K) \partial_{K} Q(K)\right] \\
& =\frac{1}{2 \pi i} \int_{-\pi}^{\pi} d K \frac{d \ln \{\operatorname{det}[Q(K)]\}}{d K} \\
& =-\frac{1}{2 \pi} \oint d \arg \{\operatorname{det}[Q(K)]\} .
\end{aligned}
$$

According to bulk-boundary correspondence, the nontrivial topological invariant in a bulk is always accompanied by the existence of topological edge states at the boundaries. Under the open boundary condition (OBC), the energy spectrum with respect to the zigzag angle is shown in Fig. 2(c). The spectrum can be either gapless or gapped, where in-gap zero-energy states appear only in the gapped case, implying topologically nontrivial properties. To confirm this, we calculate the winding number as a function of the zigzag angle; see Fig. 2(d). Indeed, in the gapless case the winding number vanishes, while in the gapped case the winding number is equal to 1 . Our results are consistent with earlier studies [18], where the topologically nontrivial phase was predicted for $|\psi-\pi / 2|<\psi_{\text {thre }}$ with the threshold value $\psi_{\text {thre }}=$ $\arcsin \left|\left(t_{\|}+t_{\perp}\right) /\left(t_{\|}-t_{\perp}\right)\right| / 2$.

We notice here that the original winding number (6) is ill defined in the gapless case. When the energy bands are gapless, the trajectory of $\operatorname{det} Q$ in the complex plane crosses the original point where its angle is ambiguous; see Figs. 3(a) and 3(b). However, a tiny perturbation without breaking the chiral symmetry does not change the topological property. To some extent, these gapless cases are effectively "trivial" because they share similar topological properties to the trivial phase and exhibit significant difference from the gapped topological phase. To fix this problem, in numerical calculations we need to shift det $Q(K)$ and redefine $v^{\prime}=v \bmod 2$ to avoid this ambiguity [18]. For completeness, we also show the energy bands and the trajectory in the complex plane for the gapped case; see Figs. 3(c) and 3(d). det $Q$ is always nonvanishing, and the winding number is 1 .

\section{B. Disordered arrays}

Next, we study the effects of disorder on the zigzag array. When the zigzag angle between nanoparticles experiences random variations, we may expect the well-known Anderson localization to occur. At the same time, in the topologically nontrivial case characterized by the winding number $v=1$, we expect topological edge states, which are localized exponentially at one or two edges. Since the zigzag-angle disorder can be realized experimentally [13], we study the interplay between topology-induced edge localization and the Anderson localization. In the following, we keep $t_{\|}=2, t_{\perp}=-1$ fixed and assume that disorder is applied to the zigzag angle, $\psi=$ $\psi_{0}+\delta \psi_{i}$, with averaged zigzag angle $\psi_{0}$ and $\delta \psi_{i}=W \epsilon_{i}$, where $\epsilon_{i}$ is a random number distributed uniformly within $[-\pi / 2, \pi / 2]$ and $W$ is dimensionless. Such disorder affects only the coupling, and it will not break the chiral symmetry. As the range of zigzag angles $\psi$ is limited [see Fig. 5(a)], the strength of random angles $\delta \psi_{i}$ should be limited within $\delta \psi \in$ $[-\pi / 2, \pi / 2]$. Thus the strength of disorder in this context is restricted to the domain $W \in[0,1]$. Based on the degree of distortion, we may say that the disorder on the zigzag angle is weak when $W \rightarrow 0$ and the disorder is strong when $W \rightarrow 1$. This can be seen from the experimentally measured data in Appendix A.

The winding number (6) is obtained generally in translation-invariant systems, and usually the straightforward definition fails for disordered systems. If disorder does not break the essential discrete symmetry, i.e., the chiral symmetry, the winding number can still be extracted via a real-space formula $[21,22]$. Calculation of the realspace winding number relies on the homotopically equivalent flat-band Hamiltonian $\hat{Q}=\hat{P}_{+}-\hat{P}_{-}$, in which

$$
\begin{aligned}
& \hat{P}_{+}=\sum_{n \in\left\{E_{n}>0\right\}}\left|\varphi_{n}\right\rangle\left\langle\varphi_{n}\right|, \\
& \hat{P}_{-}=\sum_{n \in\left\{E_{n}<0\right\}}\left|\varphi_{n}\right\rangle\left\langle\varphi_{n}\right|
\end{aligned}
$$

are the projectors onto the subspace with positive or negative energies, respectively. It can be found that $\hat{Q}$ shares the same eigenstates with the Hamiltonian $\hat{H}[20,23]$. For chiralsymmetric systems, $Q$ can be composed by $Q=Q_{A B}+Q_{B A}$, with $Q_{A B}=\Gamma_{A} Q \Gamma_{B}, Q_{B A}=\Gamma_{B} Q \Gamma_{A}$. Here, $\Gamma_{A}$ and $\Gamma_{B}$ are the projectors onto the two sublattices, labeled by $A$ and $B$, respectively. Then, the winding number $v$ can be calculated in real space via the formula $[21,24,25]$

$$
v=-\operatorname{Tr}\left\{Q_{B A}\left[X, Q_{A B}\right]\right\},
$$

where $X$ is the position operator. This formula remains valid even in the presence of disorder [21,22,24,26], and it has been used widely in studies of disorder and topology [27-31]. 

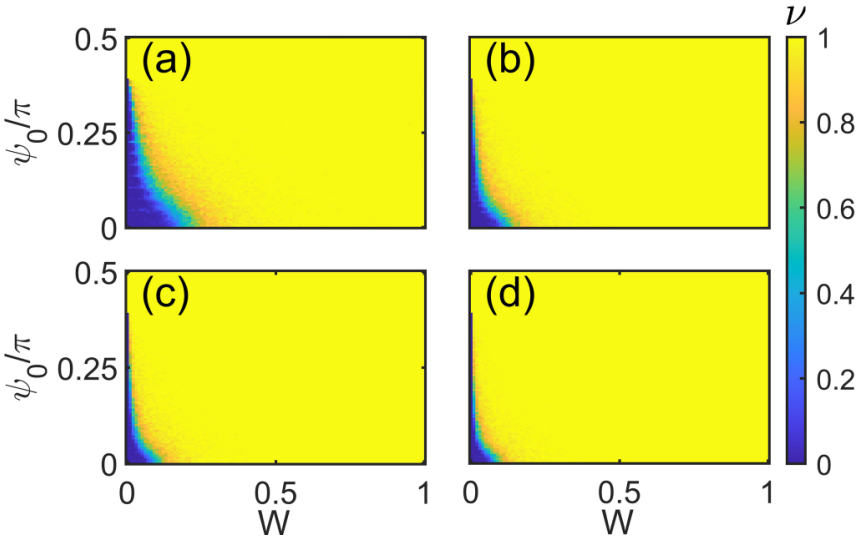

FIG. 4. Winding number for different zigzag angles $\psi_{0}$ and strength of disorder $W$. The cases (a)-(d) are calculated for 62, 122, 162, and 202 nanoparticles, respectively.

As the topological property originates from the bulk, the $\mathrm{PBC}$ is more suitable than the OBC to capture the bulk property. Besides, it is convenient to impose the PBC to avoid the effect of edge states. In this case, the position operator should be constructed carefully for the periodic boundary [32]. Therefore, in the following context, we shall use the PBC to calculate the winding number by default.

In our system, the gapless regime results in ambiguity when we calculate the winding number. However, when disorder is present, this quantity is shown to be valid even if the gap is closed $[21,33]$. We calculate the phase diagram of the winding number in the parameter space $\left(\psi_{0}, W\right)$; see Fig. 4. As it turns out, the topology of such a system is nontrivial for a large area where $W>0$. The appearance of a small area of the trivial phase can be understood as the finite-size effect, and it will tend to disappear in the thermodynamic limit $L \rightarrow \infty$. This analysis suggests that the system may become topological when disorder of the zigzag angle is added. In the regular case $(W=0)$, the gapless region $\left|\psi_{0}-\pi / 2\right|>\psi_{\text {thre }}$ is topologically trivial. Once the disorder is added, it immediately changes to topologically nontrivial. Hence the case $W=0$ can be understood as a critical point of the phase transition for the region $\left|\psi_{0}-\pi / 2\right|>\psi_{\text {thre. }}$. When disorder is present, the system is pushed towards a topological phase. This is by analogy with the metal-insulator transition induced by disorder according to Anderson's theory [6,34]. The difference is that the system is a topological Anderson insulator when disorder is present. We will discuss the property of localization in the next section.

\section{ZERO-ENERGY LOCALIZATION LENGTH}

Above, we have found that our system is topologically nontrivial when the angle disorder is included. We notice that, for one-dimensional chiral-symmetric systems, a topological transition is usually accompanied by a divergence of the localization length at $E=0[21,35,36]$. The Lyapunov exponent, which is related to the inverse of the localization length, will vanish at the point of the topological transition, and then it changes its sign. This important quantity has been used widely to probe the topological phase transition $[21,29,33]$.

\section{A. Transfer-matrix approach}

First, we start with the transfer-matrix method and employ it for our system. According to the theory of Anderson localization [6,34], a one-dimensional lattice system becomes an insulator as long as any, even weak, disorder appears, and the electron wave function decays exponentially characterizing a localized state,

$$
|\varphi(r)| \sim \exp \left(-\frac{\left|r-r_{0}\right|}{\xi}\right),
$$

where $\xi$ is called the "localization length." By employing the transfer-matrix technique [37,38], we calculate the localization length of our one-dimensional system. The discrete Schrödinger equation for the eigenstates of our model reads

$$
\begin{gathered}
t_{x}^{i, i+1} \varphi_{i}^{x}+t_{x}^{i+1, i+2} \varphi_{i+2}^{x}+t_{x y}^{i, i+1} \varphi_{i}^{y}+t_{x y}^{i+1, i+2} \varphi_{i+2}^{y}=E \varphi_{i+1}^{x}, \\
t_{y}^{i, i+1} \varphi_{i}^{y}+t_{y}^{i+1, i+2} \varphi_{i+2}^{y}+t_{x y}^{i, i+1} \varphi_{i}^{x}+t_{x y}^{i+1, i+2} \varphi_{i+2}^{x}=E \varphi_{i+1}^{y},
\end{gathered}
$$

where $\varphi^{x}$ and $\varphi^{y}$ are the wave functions of the $x$ and $y$ polarized modes at the $i$ th site, respectively. $t_{\sigma}^{i, i+1}(\sigma=x, y)$ is the coupling strength between the $i$ th and $(i+1)$ th particles,

$$
\begin{aligned}
t_{x} & \equiv t_{\|} \cos ^{2} \psi / 2+t_{\perp} \sin ^{2} \psi / 2, \\
t_{y} & \equiv t_{\|} \sin ^{2} \psi / 2+t_{\perp} \cos ^{2} \psi / 2, \\
t_{x y} & \equiv\left(t_{\|}-t_{\perp}\right) \sin \psi / 2 \cos \psi / 2 .
\end{aligned}
$$

Equation (10) can be written in a matrix form

$$
\Phi_{i+1}=M_{i} \Phi_{i}, \quad \Phi_{i}=\left(\begin{array}{c}
\varphi_{i}^{x} \\
\varphi_{i}^{y} \\
-t_{x}^{i, i+1} \varphi_{i+1}^{x}-t_{x y}^{i, i+1} \varphi_{i+1}^{y} \\
-t_{x y}^{i, i+1} \varphi_{i+1}^{x}-t_{y}^{i, i+1} \varphi_{i+1}^{y}
\end{array}\right),
$$

where the matrix $M_{i}$,

$$
M_{i}=\left(\begin{array}{cccc}
0 & 0 & t_{y} / t_{\|} t_{\perp} & -t_{x y} / t_{\|} t_{\perp} \\
0 & 0 & -t_{x y} / t_{\|} t_{\perp} & t_{x} / t_{\|} t_{\perp} \\
t_{x} & t_{x y} & E t_{y} / t_{\|} t_{\perp} & -E t_{x y} / t_{\|} t_{\perp} \\
t_{x y} & t_{y} & -E t_{x y} / t_{\|} t_{\perp} & E t_{x} / t_{\|} t_{\perp},
\end{array}\right),
$$

is the so-called transfer matrix between the $i$ th and $(i+1)$ th sites; we use the fact that $t_{x} t_{y}-t_{x y}^{2}=t_{\|} t_{\perp}$ from Eq. (10) and also apply a constraint $\operatorname{det}\left(M_{i}\right)=1$. Thus we are able to know the $L$ th wave function of the entire system by multiplying iteratively the transfer matrices,

$$
\Phi_{L}=\prod_{i=1}^{L-1} M_{i} \Phi_{1} .
$$

The Lyapunov exponent $\lambda$, which is the inverse of localization length $\lambda \equiv 1 / \xi$, can be calculated from the eigenvalue problem

$$
\mathcal{M}=\left[\lim _{L \rightarrow \infty}\left(\prod_{i=1}^{L} M_{i}\right)\left(\prod_{i=1}^{L} M_{i}\right)^{\dagger}\right] .
$$



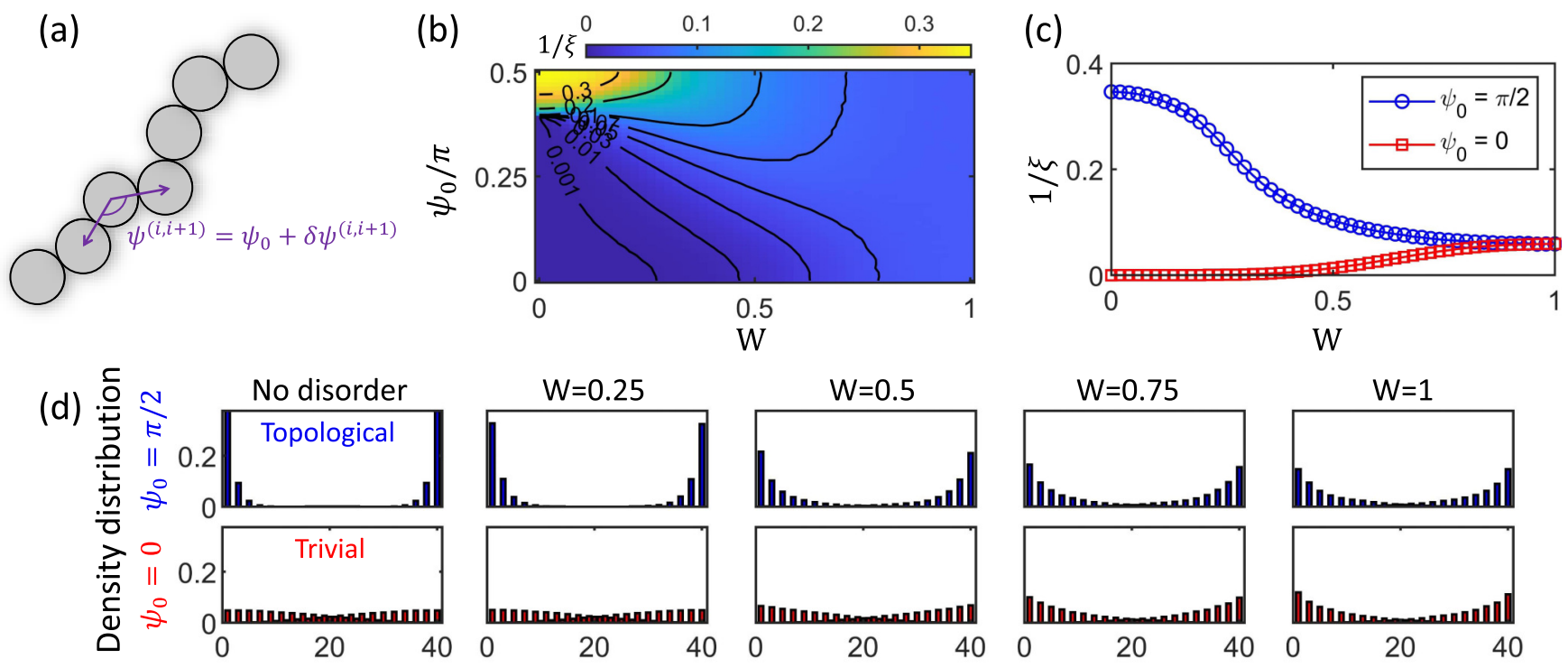

Particle index in the array

FIG. 5. (a) Schematic of disordered zigzag array. (b) Inverse localization length $1 / \xi$, obtained from the transfer-matrix method, vs the disorder strength $W$ and the zigzag angle $\psi_{0}$. Black lines are the contours of $1 / \xi$. Due to the symmetry about $\psi_{0}=\pi / 2$, we only show the results of $\psi_{0} \in[0, \pi / 2]$. (c) The inverse localization length of $\psi_{0}=\pi / 2,0$ vs disorder strength $W$. (d) Density distributions of the eigenstates which are the closest to $E=0$ for different disorder strengths. Upper and lower panels correspond to $\psi_{0}=\pi / 2$ and $\psi_{0}=0$, respectively. In our calculations, we choose 40 particles and average over 500 random realizations. The density of each particle is the summation of the squared absolute value of the $x$ - and $y$-mode wave function.

Here, we employ the efficient numerical method of Refs. $[39,40]$ to calculate the localization length.

\section{B. Topology and localization length}

In Fig. 5(b), we show the phase diagram of the inverse localization length $1 / \xi$ with respect to $\left(W, \psi_{0}\right)$ at $E=0$. In the gapped case, the zero-energy localization length is exactly the length of localization of the edge state. Disorder will increase the localization length; see the blue curve in Fig. 5(c). In the gapless case, the zero-energy localization length diverges, and disorder will result in a decrease of the localization length; see the red curve in Fig. 5(c). We find that divergence of the localization length occurs only in the gapless region $\left|\psi_{0}-\pi / 2\right|>\psi_{\text {thre }}$ when $W=0$, and we do not find any divergence of the localization length when $W>0$. This implies that the system remains in the same topological phase apart from the region $\left(\left|\psi_{0}-\pi / 2\right|>\psi_{\text {thre }}, W=0\right)$. Thus we may conclude that the topologically nontrivial system remains topological when $W>0$.

As we find that the winding number of the system remains nontrivial for $W>0$, the system should present topologically nontrivial phenomena. One of the features in one-dimensional topological systems is the appearance of topological edge states [41]. In Fig. 5(d), we show the density distribution of the eigenstates with $E \approx 0$ under different strengths of disorder. We find that the topological edge states with averaged zigzag angle $\psi_{0}=\pi / 2$ are preserved as the value of $W$ grows. Meanwhile, with an increase of disorder strength, there appear localized states for the zigzag angle $\psi_{0}=0$. For a weak disorder $W \approx 0$, the localization length of the edge state is so large that the edge state is difficult to distinguish for a small number of nanoparticles. However, the edge-localization effect will become distinct when the system size is increased. We would like to emphasize that when the system is topological, the edge state will appear in each random realization; see Appendix B for more information.

\section{Experimental results}

We have verified our theoretical predictions experimentally. For the experiment, we fabricate many different types of disordered zigzag arrays of nanodisks placed on a glass substrate, as shown in Figs. 6(a)-6(d). The samples are obtained with the same platform and techniques as described earlier in Ref. [13]. To map topological states, we excite zigzag arrays by femtosecond laser pulses and observe the generation of a third-harmonic signal, this being a tool for mapping strongly spatially localized distributions of light in the arrays. We set different strengths of the angle disorder, as shown in the examples in Figs. 6(a)-6(d) and also in Appendix A, Fig. 10. We analyze the edge localization via the third-harmonic field in the zigzag arrays composed of 11 nanodisks with the averaged zigzag angle $\psi_{0}=\pi / 2$ and different angle disorder. We find that the number of distinguishable edge states decreases when the disorder strength increases [see Fig. 6(e)]; however, some of the edge is clearly observed for most of the cases. The reduction in the edge localization observed experimentally corresponds directly to the effect predicted numerically, and it can be understood as follows. The localization length increases with the strength of disorder $W$ for the averaged zigzag angle $\psi_{0}=\pi / 2$; see Fig. $5(\mathrm{~d})$. 
(a) $\delta \psi=5^{\circ}$

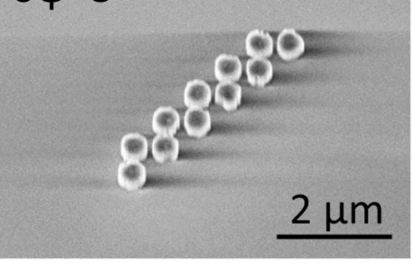

(b)

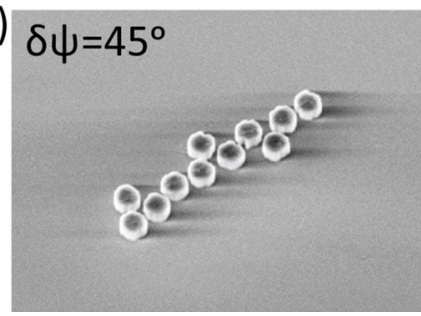

(c) $\delta \psi=60^{\circ}$

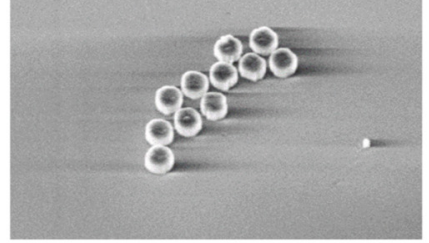

(d) $\delta \psi=90^{\circ}$

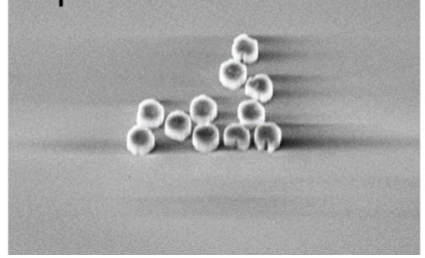

(e)

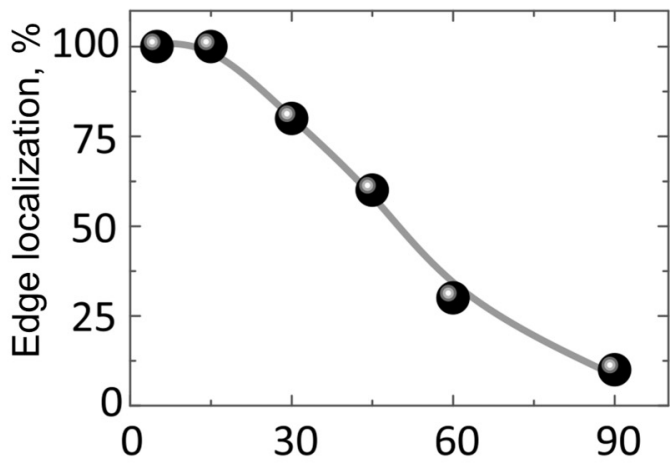

Disorder angle, degree

FIG. 6. Experimental results for disordered nanoparticle arrays. (a)-(d) Scanning electron microscopy (SEM) image of fabricated zigzag arrays of Mie-resonant dielectric nanodisks with disordered strengths $\delta \psi=5^{\circ}, 45^{\circ}, 60^{\circ}$, and $90^{\circ}$, respectively. The averaged zigzag angle is $\psi_{0}=\pi / 2$, which is the same setup as in Ref. [13]. (e) Edge-localization ratio extracted from experimental observations as a function of disorder angle.

When the localization length of the edge state exceeds the length of the array, we are not able to distinguish it from other states, as similarly observed for the trivial case when $\psi_{0}=0$; see Fig. 5(d). For example, the localization length of the edge state is about $\xi=10$ at $W=0.5$ (this corresponds to the disorder angle $\delta \psi \approx \pi / 4$ ). This is almost the same length as the extension of the 11-disk nanoparticle arrays employed in our experiment. Thus, while in general we confirm the basic prediction of the theory about the character of the topologyinduced localization and its interplay with the disorder, it becomes difficult to recognize the edge states and their localization for stronger disorder when $W>0.5$ with finite-extent arrays.

\section{STAGGERED-COUPLING ZIGZAG ARRAYS}

Above, we have found theoretically that disorder-induced topological transitions may happen only in the gapless case when $\left|\psi_{0}-\pi / 2\right|>\psi_{\text {thre }}$. Here, we consider a generalized model, explore richer topological phases, and predict disorder-induced topological phase transitions. We notice that the coupling between particles strongly depends on the distance between nanoparticles [12], and in our model discussed above all nanoparticles are equally spaced. In this section, we consider a staggered-spacing array in which the relative distance between nanoparticles alternates for odd and even bonds, as shown in Fig. 7(a). In this case, the generalized Hamiltonian reads

$$
\hat{H}_{2}=\sum_{j, v, v^{\prime}}\left(1+\Delta^{(j)}\right) a_{j, v}^{\dagger} V_{\nu, v^{\prime}}^{(j+1)} a_{j+1, v^{\prime}}+\text { H.c. },
$$

where $\Delta^{(j)}=0$ for an even bond and $\Delta^{(j)}=\Delta$ for an odd bond.

\section{A. Ideal arrays}

We start with an ideal array when disorder is absent. Chiral symmetry is still preserved in the presence of the extra term, and the topological property is still characterized by the winding number equation (6). The phase diagram of the winding

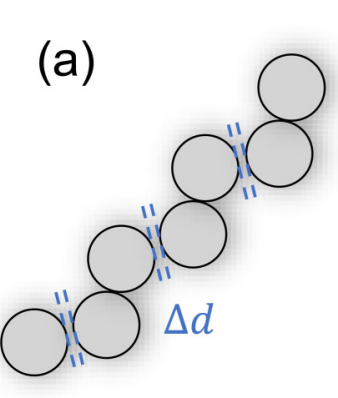

(c)

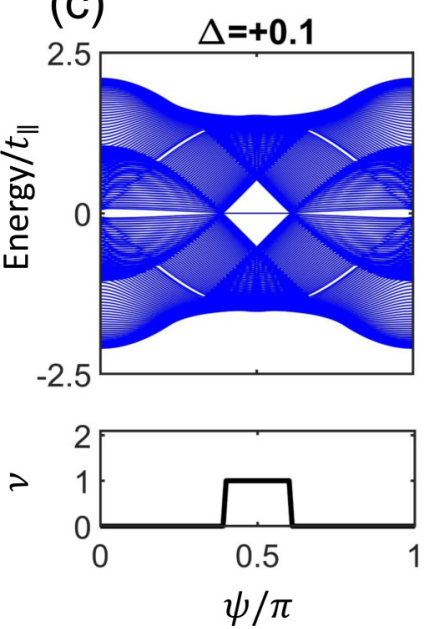

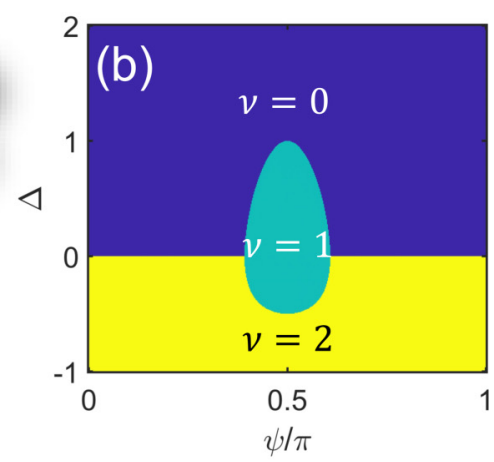

(d)
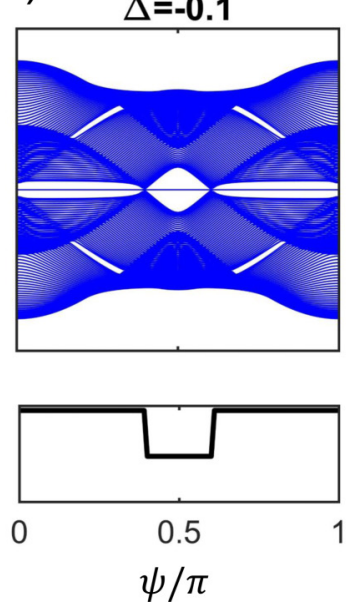

FIG. 7. (a) Schematic of a staggered-spacing zigzag array. $\Delta d$ indicates a larger (or less) space at the odd bond. (b) Phase diagram of winding number $v$ with respect to the bias $\Delta$ and the zigzag angle $\psi$. (c) and (d) Two typical energy spectra under the OBC and the corresponding winding number vs the zigzag angle. The winding number is determined from Eq. (6). 

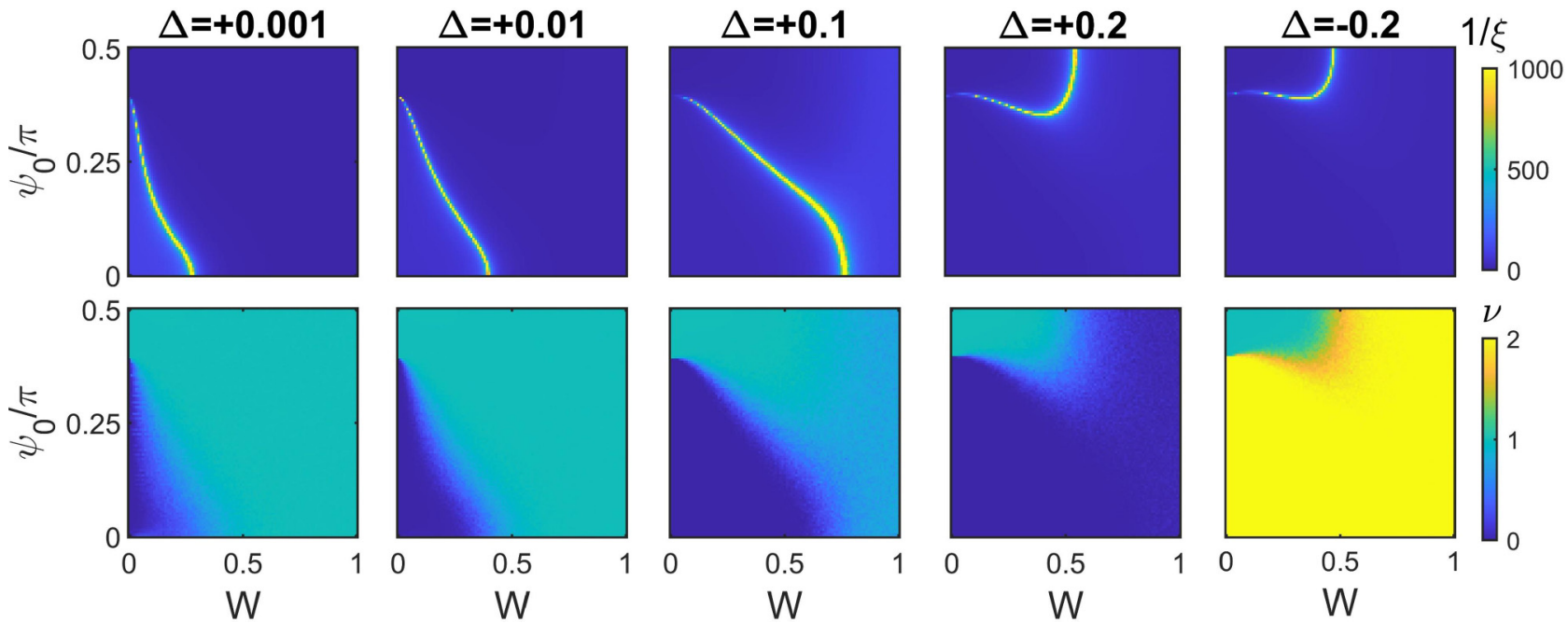

FIG. 8. Top row: Localization length. Calculation of the transfer matrix iterated at least $10^{6}$ times. Bottom row: Winding number. Change of the topological transition boundary for $\Delta<0$ is similar to $\Delta$, and thus we have omitted other cases for simplicity. In our calculations, we use 202 particles under the PBC and average over 200 random realizations.

number is obtained by varying the averaged zigzag angle and disorder strength; see Fig. 7(b). We uncover three topologically different phases characterized by the winding numbers $v=0,1,2$. When $\Delta=0$, the generalized model becomes the original model of the zigzag array discussed above.

In Figs. 7(c) and 7(d), we show two typical energy spectra under the $\mathrm{OBC}$ and the corresponding winding numbers. When there is no zero-energy edge mode, the topological phase is trivial, and therefore $v=0$. When zero-energy edge modes appear, the zero-energy edge states are degenerate, and their numbers $n_{\text {edge }}=2$ and $n_{\text {edge }}=4$ correspond to $v=1$ and $v=2$, respectively. This reflects the well-known bulkedge correspondence.

\section{B. Disordered arrays}

Next, we study the effects of disorder in zigzag angles. In Fig. 8, we show the phase diagram of the localization length in the parameter space $\left(\psi_{0}, W\right)$ as $\Delta$ changes (see the top row). For each fixed $\Delta$, we find a narrow yellow line in the diagram with an extremely large localization length, indicating a boundary of a topological phase transition. We also calculate the phase diagram of the winding number using Eq. (8), as shown in the bottom row in Fig. 8. The boundary of the phase transition coincides with the one shown in the diagram of the localization length. Besides, we notice that the region of trivial winding numbers shrinks to $W=0$ when $\Delta$ vanishes. This is consistent with the case of $\Delta=0$ discussed above, where the system is topologically nontrivial in the presence of angle disorder for any $W>0$.

As stated above, we observe three topologically distinct phases characterized by $v=0,1,2$. Besides the disorderinduced topological transition between $v=0$ and $v=1$, there is also the transition between $v=1$ and $v=2$ when $\Delta<0$. The phase diagram of $\Delta<0$ is similar to that of $\Delta>0$; see the last column in Fig. 8. Next, we compare two examples of $\Delta>0$ and $\Delta<0$ in Fig. 9. We identify a disappearance of twofold degenerate edge states in Fig. 9(a), corresponding to the transition from $v=1$ to $v=0$. In Fig. 9(b), we find that twofold degenerate edge states are transformed into fourfold degenerate edge states, which characterizes the transition from $v=1$ to $v=2$. A change in the number of zero-energy edge states is a result of a topological transition, and the number of zero-energy edge states coincides with the winding number in Figs. 9(c) and 9(d). (a) $\Delta=+0.3$
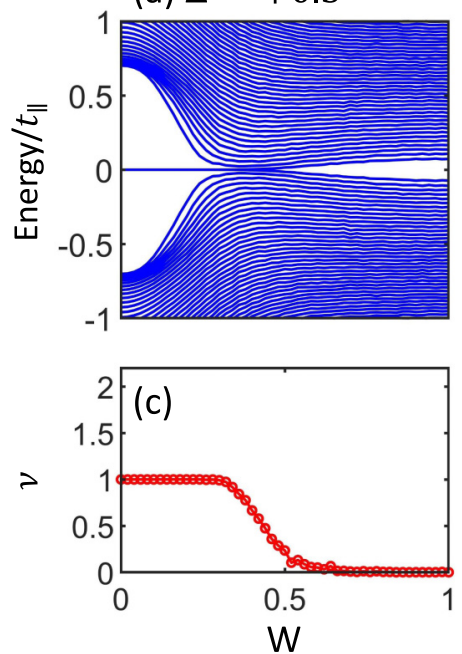

(b) $\Delta=-0.3$
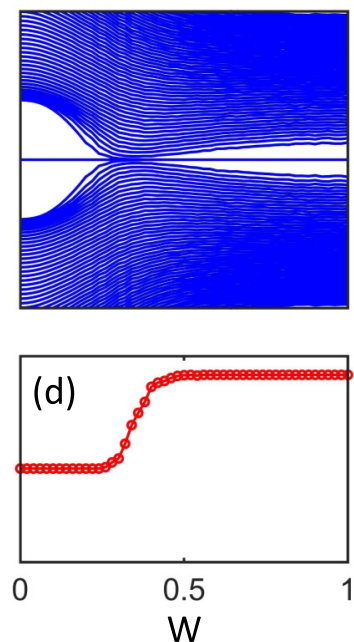

FIG. 9. (a) and (b) Energy spectrum with respect to the strength of disorder $W$ near $E=0$ with $\Delta=+0.3$ and $\Delta=-0.3$, respectively. Here, the averaged zigzag angle is $\psi_{0}=\pi / 2$. Zero-energy edge modes in (a) are twofold degenerate. Zero-energy edge modes in (b) are twofold degenerate in the clean limit $W=0$ and then turn into fourfold degenerate after the phase transition. (c) and (d) are the winding numbers of (a) and (b), respectively. All results are averaged over 200 random realizations. 
When the deviation $|\Delta|$ is small, the spectral gap will be closed when disorder becomes stronger. Intuitively, this happens because the disorder becomes dominating, and it "wipes away" the details of the staggered-spacing distribution. However, in both these cases in Fig. 9, we observe that the spectral gap does not vanish for a strong disorder. This result differs from the common belief that stronger disorder will force a spectral gap to close [21] or topological transitions occur due to disappearance of energy gaps. Our finding may help to better understand the nature of disorder-induced topological transitions.

\section{CONCLUSIONS}

We have studied the effect of disorder on topological properties of zigzag arrays of nanoparticles with polarizationdependent interaction. For equal-spacing arrays, we have found that the system will remain in the topological regime even in the presence of an angle disorder supporting highly or weakly localized edge states, and this feature was found to be in agreement with experiments for finite-extent arrays of silicon nanoparticles with an introduced disorder. For staggered-spacing arrays, we have found richer topological phases and observed that the angle disorder may induce topological phase transitions. Remarkably, in this latter case the spectral gap remains open even for a strong disorder, and we believe this system provides an example of a topologically nontrivial system with disordered parameters.

\section{ACKNOWLEDGMENTS}

This work has been supported by the Key-Area Research and Development Program of Guangdong Province under Grant No. 2019B030330001, the National Natural Science Foundation of China (NNSFC) under Grants No. 11874434 and No. 11574405, and the Science and Technology Program of Guangzhou (China) under Grant No. 201904020024. The research of Y.K. is supported by the Office of China Postdoctoral Council (Grant No. 20180052), the National Natural Science Foundation of China (Grant No. 11904419), and the Australian Research Council (Grant No. DP200101168).

\section{APPENDIX A: DETAILED EXPERIMENTAL DATA}

We present in Fig. 10 results on experimentally accumulated statistics of field distributions in zigzag chains with angular disorder varying from $\delta \psi=5^{\circ}$ to $\delta \psi=90^{\circ}$. All chains are 11 disks long, and their configurations are schematically shown in the left-hand columns. The field distributions along the chains are studied via nonlinear imaging: The chains are excited by short-pulse, high-peak-power laser pulses at 1590-nm wavelength, and generation of a third-harmonic

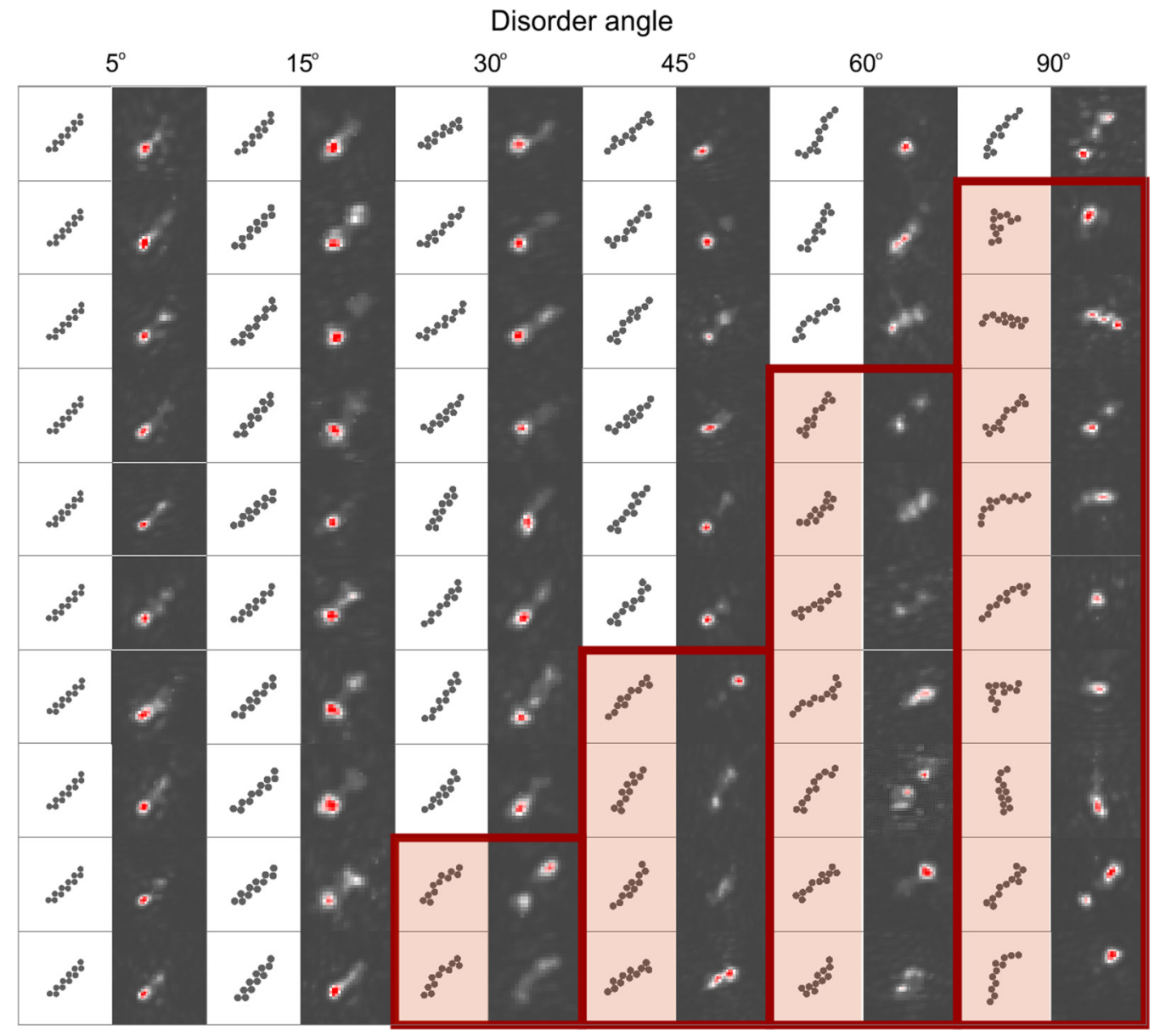

FIG. 10. Experimentally observed field distributions in 60 realizations of disordered zigzag arrays illuminated by linearly polarized light. 
signal is imaged onto a camera. The corresponding distributions of the third harmonic are shown as false-color images in the right-hand columns. In the chosen experimental configuration, the topological state is expected to occur at the bottom left edge of the chains. Experimental realizations in which the formation of the edge state was not observed are highlighted in red. As the level of disorder increases, the localization length of the edge states increases, reaching and further surpassing the length of the 11-disk chains. Large localization length in highly disordered zigzags reduces the probability of observing edge states in finite-length chains.

\section{APPENDIX B: EDGE STATE IN SINGLE REALIZATION}

We have mentioned in the main text that when a system is in a topologically nontrivial phase, there appears an edge state with zero energy. This can be accounted for by the bulk-edge correspondence principle. To show this, we present the energy spectrum as a function of disorder strength $W$ in single random realization $\left\{\epsilon_{i}\right\}$; see Fig. 11 . We calculate the edge occupation $n_{\text {edge }}=\sum_{l \in \text { edge }} n_{l}$ to examine if the zero-energy state is localized around the edge. The results show that the zero-energy state is indeed an edge state. This implies that the bulk-edge correspondence principle applies for each random realization if the system is in a topological phase. (a)
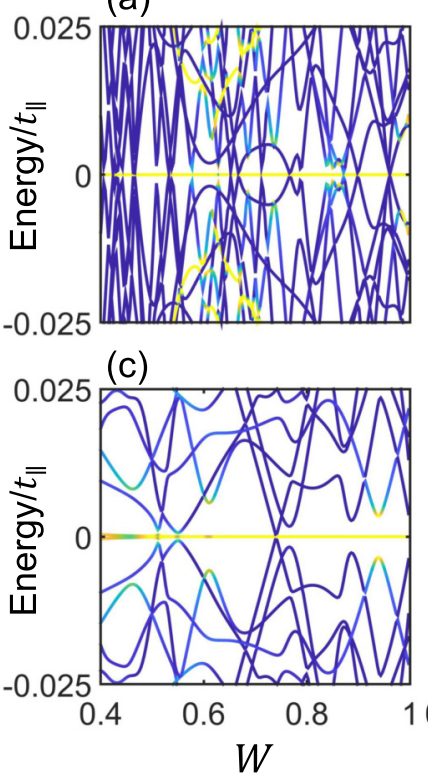

(b)

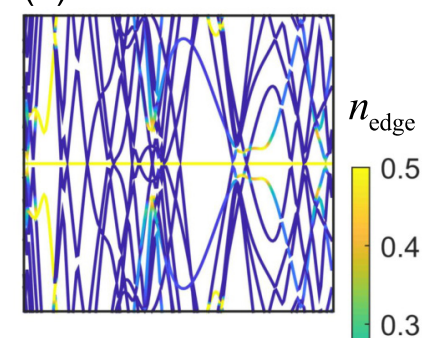

(d)

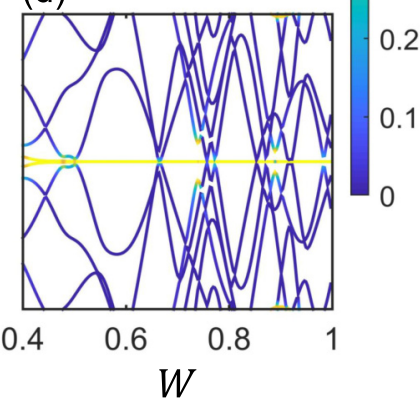

FIG. 11. Energy spectrum as a function of disorder strength $W$ in a single realization $\left\{\epsilon_{i}\right\}$. (a) and (b) show two different random realizations when $\psi_{0}=\pi / 2$, and (c) and (d) show two different random realizations when $\psi_{0}=0$. The length of the system is 402 particles, and the area of the edge is set to be the leftmost and rightmost 50 particles. Other parameters are the same as in Fig. 5.

[1] L. Lu, J. D. Joannopoulos, and M. Soljačić, Topological states in photonic systems, Nat. Phys. 12, 626 (2016).

[2] T. Ozawa, H. M. Price, A. Amo, N. Goldman, M. Hafezi, L. Lu, M. C. Rechtsman, D. Schuster, J. Simon, O. Zilberberg, and I. Carusotto, Topological photonics, Rev. Mod. Phys. 91, 015006 (2019).

[3] P. St-Jean, V. Goblot, E. Galopin, A. Lemaître, T. Ozawa, L. Le Gratiet, I. Sagnes, J. Bloch, and A. Amo, Lasing in topological edge states of a one-dimensional lattice, Nat. Photonics 11, 651 (2017).

[4] S. Barik, A. Karasahin, C. Flower, T. Cai, H. Miyake, W. DeGottardi, M. Hafezi, and E. Waks, A topological quantum optics interface, Science 359, 666 (2018).

[5] S. Mukherjee and M. C. Rechtsman, Observation of Floquet solitons in a topological bandgap, Science 368, 856 (2020).

[6] P. W. Anderson, Absence of diffusion in certain random lattices, Phys. Rev. 109, 1492 (1958).

[7] F. Riboli, N. Caselli, S. Vignolini, F. Intonti, K. Vynck, P. Barthelemy, A. Gerardino, L. Balet, L. H. Li, A. Fiore, M. Gurioli, and D. S. Wiersma, Engineering of light confinement in strongly scattering disordered media, Nat. Mater. 13, 720 (2014).

[8] Z. Daozhong, H. Wei, Z. Youlong, L. Zhaolin, C. Bingying, and Y. Guozhen, Experimental verification of light localization for disordered multilayers in the visible-infrared spectrum, Phys. Rev. B 50, 9810 (1994).

[9] H. Hu, A. Strybulevych, J. Page, S. E. Skipetrov, and B. A. van Tiggelen, Localization of ultrasound in a three-dimensional elastic network, Nat. Phys. 4, 945 (2008).
[10] J. Billy, V. Josse, Z. Zuo, A. Bernard, B. Hambrecht, P. Lugan, D. Clément, L. Sanchez-Palencia, P. Bouyer, and A. Aspect, Direct observation of Anderson localization of matter waves in a controlled disorder, Nature 453, 891 (2008).

[11] M. Pasienski, D. McKay, M. White, and B. DeMarco, A disordered insulator in an optical lattice, Nat. Phys. 6, 677 (2010).

[12] A. Poddubny, A. Miroshnichenko, A. Slobozhanyuk, and Y. Kivshar, Topological Majorana states in zigzag chains of plasmonic nanoparticles, ACS Photonics 1, 101 (2014).

[13] S. Kruk, A. Poddubny, D. Smirnova, L. Wang, A. Slobozhanyuk, A. Shorokhov, I. Kravchenko, B. LutherDavies, and Y. Kivshar, Nonlinear light generation in topological nanostructures, Nat. Nanotechnol. 14, 126 (2019).

[14] J. W. You, Z. Lan, Q. Bao, and N. C. Panoiu, Valley-Hall topological plasmons in a graphene nanohole plasmonic crystal waveguide, IEEE J. Sel. Top. Quantum Electron. 26, 1 (2020).

[15] Q. Cheng, Y. Pan, Q. Wang, T. Li, and S. Zhu, Topologically protected interface mode in plasmonic waveguide arrays, Laser Photonics Rev. 9, 392 (2015).

[16] J. W. You, Z. Lan, and N. C. Panoiu, Four-wave mixing of topological edge plasmons in graphene metasurfaces, Sci. Adv. 6, eaaz3910 (2020).

[17] S. Yves, R. Fleury, T. Berthelot, M. Fink, F. Lemoult, and G. Lerosey, Crystalline metamaterials for topological properties at subwavelength scales, Nat. Commun. 8, 1 (2017).

[18] A. P. Slobozhanyuk, A. N. Poddubny, A. E. Miroshnichenko, P. A. Belov, and Y. S. Kivshar, Subwavelength Topological Edge States in Optically Resonant Dielectric Structures, Phys. Rev. Lett. 114, 123901 (2015). 
[19] S. Kruk, A. Slobozhanyuk, D. Denkova, A. Poddubny, I. Kravchenko, A. Miroshnichenko, D. Neshev, and Y. Kivshar, Edge states and topological phase transitions in chains of dielectric nanoparticles, Small 13, 1603190 (2017).

[20] S. Ryu, A. P. Schnyder, A. Furusaki, and A. W. Ludwig, Topological insulators and superconductors: Tenfold way and dimensional hierarchy, New J. Phys. 12, 065010 (2010).

[21] I. Mondragon-Shem, T. L. Hughes, J. Song, and E. Prodan, Topological Criticality in the Chiral-Symmetric AIII Class at Strong Disorder, Phys. Rev. Lett. 113, 046802 (2014).

[22] E. Prodan and H. Schulz-Baldes, Non-commutative odd Chern numbers and topological phases of disordered chiral systems, J. Funct. Anal. 271, 1150 (2016).

[23] C.-K. Chiu, J. C. Y. Teo, A. P. Schnyder, and S. Ryu, Classification of topological quantum matter with symmetries, Rev. Mod. Phys. 88, 035005 (2016).

[24] E. J. Meier, F. A. An, A. Dauphin, M. Maffei, P. Massignan, T. L. Hughes, and B. Gadway, Observation of the topological Anderson insulator in disordered atomic wires, Science $\mathbf{3 6 2}$, 929 (2018).

[25] E. Prodan and H. Schulz-Baldes, Bulk and Boundary Invariants for Complex Topological Insulators (Springer, New York, 2016).

[26] H. Schulz-Baldes, Topological insulators from the perspective of non-commutative geometry and index theory, Jahresber. Dtsch. Math.-Ver. 118, 247 (2016).

[27] J. K. Asbóth, B. Tarasinski, and P. Delplace, Chiral symmetry and bulk-boundary correspondence in periodically driven onedimensional systems, Phys. Rev. B 90, 125143 (2014).

[28] C.-B. Hua, R. Chen, D.-H. Xu, and B. Zhou, Disorder-induced Majorana zero modes in a dimerized Kitaev superconductor chain, Phys. Rev. B 100, 205302 (2019).

[29] J. Claes and T. L. Hughes, Disorder driven phase transitions in weak AIII topological insulators, Phys. Rev. B 101, 224201 (2020).
[30] M. Maffei, A. Dauphin, F. Cardano, M. Lewenstein, and P. Massignan, Topological characterization of chiral models through their long time dynamics, New J. Phys. 20, 013023 (2018).

[31] A. Haim and A. Stern, Benefits of Weak Disorder in OneDimensional Topological Superconductors, Phys. Rev. Lett. 122, 126801 (2019)

[32] R. Resta, Quantum-Mechanical Position Operator in Extended Systems, Phys. Rev. Lett. 80, 1800 (1998).

[33] J. Song and E. Prodan, AIII and BDI topological systems at strong disorder, Phys. Rev. B 89, 224203 (2014).

[34] F. Evers and A. D. Mirlin, Anderson transitions, Rev. Mod. Phys. 80, 1355 (2008).

[35] A. R. Akhmerov, J. P. Dahlhaus, F. Hassler, M. Wimmer, and C. W. J. Beenakker, Quantized Conductance at the Majorana Phase Transition in a Disordered Superconducting Wire, Phys. Rev. Lett. 106, 057001 (2011).

[36] I. C. Fulga, F. Hassler, A. R. Akhmerov, and C. W. J. Beenakker, Scattering formula for the topological quantum number of a disordered multimode wire, Phys. Rev. B 83, 155429 (2011).

[37] J. T. Chalker and M. Bernhardt, Scattering Theory, Transfer Matrices, and Anderson Localization, Phys. Rev. Lett. 70, 982 (1993).

[38] C. W. J. Beenakker, Random-matrix theory of quantum transport, Rev. Mod. Phys. 69, 731 (1997).

[39] A. Mackinnon and B. Kramer, The scaling theory of electrons in disordered solids: Additional numerical results, Z. Phys. B: Condens. Matter 53, 1 (1983).

[40] A. MacKinnon and B. Kramer, One-Parameter Scaling of Localization Length and Conductance in Disordered Systems, Phys. Rev. Lett. 47, 1546 (1981).

[41] G. M. Graf and J. Shapiro, The bulk-edge correspondence for disordered chiral chains, Commun. Math. Phys. 363, 829 (2018). 